\title{
Estimating Transmission Line Parameters of Three-core Power Cables with Common Earth Screen
}

\author{
Yan $\mathrm{Li}$, \\ Peter A. A. F. Wouters \\ Eindhoven University of Technology, \\ the Netherlands
}

\begin{abstract}
The propagation modes of travelling wave in a threecore power cable (XLPE or PILC) with common earth screen can be uncoupled into three modes: one shield to phase (SP) mode and two phase to phase (PP) modes. A generic model approach is applied in this paper to estimate the transmission line parameters of three-core cables with common earth screen, which includes the effect of different layers on series impedance and admittance. The characteristic impedance, the propagation velocity and the attenuation for SP and PP modes of two cable types, PILC and XLPE are modeled and compared with test results. Calculated parameters match within $5 \%$ for the characteristic impedances of different modes and 5\% for the velocity for the PILC cable. Larger deviations occurred for XLPE cable, due to difficulties in appropriate modeling the semiconducting insulation screens around each conductor. The attenuation for both PILC and XLPE is hard to predict because data on complex permittivity, being the dominant factor, is hardly known.
\end{abstract}

\section{Introduction}

High frequency signals, e.g. coming from partial discharges (PDs), behave as travelling waves when they propagate along power cables. For three-core power cable with common earth screen, three uncoupled propagation modes are observed: one shield to phase (SP) mode and two equaled phase to phase (PP) modes $[1,2]$. A mathematical model based on references $[3,4]$ is investigated to estimate the transmission line parameters (characteristic impedance, propagation velocity and attenuation) of both PILC and XLPE threecore cables for the different modes. The approach in $[3,4]$ can incorporate different parallel layers in both earth screen and inner conductor (e.g. semi-conducting layer) for the calculation. However, it is strictly only valid for cylindrical symmetric configurations. The presented modeling aims to investigate the accuracy when "effective" parameters are introduced for the essentially not rotational symmetric PILC or XLPE 3phase cables.

This paper is organized as follows: section 2 gives an overview of the mathematical model; section 3 utilizes the model for PILC cable and compares the model with test result; section 4 applies the model to XLPE cable.

\author{
Paul Wagenaars, \\ Peter C. J. M. van der Wielen, \\ E. Fred Steennis \\ DNV KEMA Energy \& Sustainability
}

\section{Series impedance and admittance model}

This section gives an overview of the model to calculate the transmission line parameters of three-core cables. The impedance and admittance are defined based on the telegraph equation.

$$
\left\{\begin{array}{l}
-\frac{\mathrm{d} \mathbf{U}}{\mathrm{d} x}=\mathbf{Z I} \\
-\frac{\mathrm{d} \mathbf{I}}{\mathrm{d} x}=\mathbf{Y U}
\end{array}\right.
$$

where $\mathbf{U}$ and $\mathbf{I}$ are vectors of the voltages and currents along a cable at distance $x . \mathbf{Z}$ and $\mathbf{Y}$ are square matrices of the impedance and admittance. For SP and PP modes of three-core cables, the impedance and admittance will reduce to $3 \times 3$ matrices [1].

$$
\mathbf{Z}=\left[\begin{array}{ccc}
Z_{s} & Z_{m} & Z_{m} \\
Z_{m} & Z_{s} & Z_{m} \\
Z_{m} & Z_{m} & Z_{s}
\end{array}\right] \quad \mathbf{Y}=\left[\begin{array}{ccc}
Y_{s} & Y_{m} & Y_{m} \\
Y_{m} & Y_{s} & Y_{m} \\
Y_{m} & Y_{m} & Y_{s}
\end{array}\right]
$$

The subscript "s" indicates self-impedance/admittance and " $m$ " refers to mutual impedance/admittance. The parameters of SP and PP modes can be expressed as [5]:

$$
\begin{aligned}
& Z_{s p}=\frac{1}{3} \sqrt{\frac{Z_{s}+2 Z_{m}}{Y_{s}+2 Y_{m}}} \\
& Z_{p p}=2 \sqrt{\frac{Z_{s}-2 Z_{m}}{Y_{s}-2 Y_{m}}} \\
& \gamma_{s p}=\sqrt{\left(Z_{s}+2 Z_{m}\right)\left(Y_{s}+2 Y_{m}\right)} \\
& \gamma_{p p}=\sqrt{\left(Z_{s}-Z_{m}\right)\left(Y_{s}-Y_{m}\right)}
\end{aligned}
$$

The impedance and admittance can be expressed with geometric and material parameters as [3]:

$$
\begin{gathered}
\mathbf{Z}=\mathbf{Z}_{\mathbf{i}}+\mathbf{Z}_{\mathbf{p}}+\mathbf{Z}_{\mathbf{c}}+\mathbf{Z}_{\mathbf{o}} \\
\mathbf{Y}=j \omega \mathbf{P}^{-1} \quad \mathbf{P}=\mathbf{P}_{\mathbf{i}}+\mathbf{P}_{\mathbf{p}}+\mathbf{P}_{\mathbf{c}}+\mathbf{P}_{\mathbf{o}}
\end{gathered}
$$

where $\mathbf{P}$ is the square matrix of "potential coefficients" [3]; the subscript " $\mathrm{i}$ " relates to single core (SC) cable internal part; "p" relates to earth screen internal part; "c" indicates the part between inner and outer surface of earth screen (the mutual effect between inner and outer surface of the screen); "o" is for the earth return path. For SP and PP modes considered in this paper, the earth return path is not relevant. Thus impedance and potential coefficient with subscript "o" are eliminated from the model. Furthermore, infinite thickness of the earth screen is assumed [3]. Thus impedance and potential coefficient with subscript "c" are also omitted in the model. In the appendix detailed formulas of $\mathbf{Z}$ and $\mathbf{P}$ are given, which have been derived for cylindrical symmetric situations. 


\section{PILC cable}

In order to verify the model, transmission line parameters measurement based on pulse injection and reflection as proposed in [1] was performed for a $12.5 \mathrm{kV} / 12.5 \mathrm{kV} 3 \times 95 \mathrm{~mm}^{2}$ PILC cable. The schematic of the cable is shown in Fig. 1. The dimensions and material data are provided in Table 1.

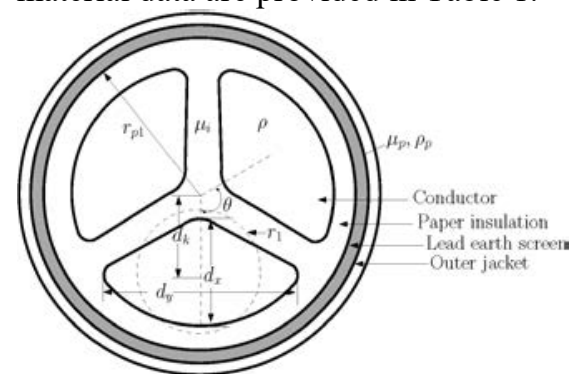

Fig. 1 - Schematic cross-section of PILC cable

Table 1 - parameters of PILC cable

\begin{tabular}{|l|l|l|l|l|}
\hline$\rho(\Omega \cdot \mathrm{m})$ & $\rho_{p}(\Omega \cdot \mathrm{m})$ & $r_{1}(\mathrm{~mm})$ & $d_{k}(\mathrm{~mm})$ & $r_{p 1}(\mathrm{~mm})$ \\
\hline $1.68 \times 10^{-8}$ & $2.20 \times 10^{-8}$ & 6.5 & 10.4 & 20.7 \\
\cline { 1 - 2 }$\varepsilon_{p}[6]$ & $\theta$ & \multicolumn{4}{|l}{} \\
\cline { 1 - 2 } $3.5-0.1 \mathrm{j}$ & $\pi / 3$ & \multicolumn{1}{|l}{} \\
\cline { 1 - 2 } & &
\end{tabular}

As a first approach $r_{l}$ (effective radius of conductor) is taken half the average of $d_{x}$ and $d_{y} ; d_{k}$ is half of $r_{p 1}$ (radius of the insulation). The measured and modeled characteristic impedance, propagation velocity, attenuation for both PP and SP mode are compared in Fig. 2, where legends with "1" indicate this approach. The characteristic impedance systematically deviates $10 \%$ for PP and $13 \%$ for SP mode. Deviation is due to non-cylindrical shape of the conductor and its positioning. Velocity and attenuation relate mainly to the characteristics of the dielectric material, in particular the value of the complex permittivity. Deviation of velocity is within $5 \%$ and $30 \%$ for attenuation. The latter deviation is related to the assumed values of the imaginary part of the permittivity. To improve the accuracy of the predicted characteristic impedance, $r_{1}$ and $d_{k}$ are optimized to match the measured values. With $r_{1} 6.2 \mathrm{~mm}$ and $d_{k} 11.4 \mathrm{~mm}$, best fitting of impedance is achieved. The measured characteristic impedances vary within $5 \%$ and $6 \%$ for the PP and the SP mode, respectively. Results are indicated with "2" in the legends in Fig. 2. The velocity mainly depends on the real permittivity of the insulation which is unaltered, giving rise to approximately equal values for both propagation modes independent of the electrode configuration. The minor change in attenuation suggests that the geometry does not affect much this parameter and simulation shows that major contribution to attenuation is related to dielectric losses.

\section{XLPE cable}

For the cable shown in Fig. $3(10 \mathrm{kV} \mathrm{XLPE}), r_{1}$ is the conductor radius; $r_{2}$ is the radius of the conductor screen; $r_{3}$ is the radius of the insulation; $r_{4}$ is the radius of the insulation screen; $r_{p 1}$ is the radius of inner swelling tape; $r_{p 2}$ is the radius of inner earth screen consisting of copper wires; $r_{p 3}$ is the inside radius of outer jacket; $r_{p 4}$ is the outside radius of the outer jacket.
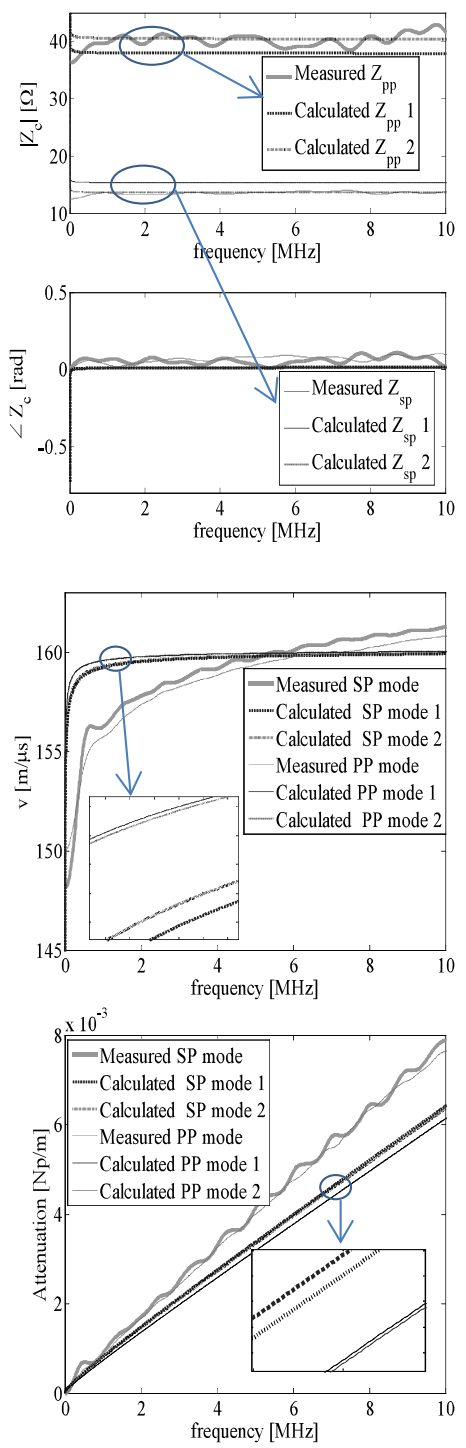

Fig. 2 - Comparison of characteristic impedance, propagation velocity, attenuation for PP and SP mode of PILC cable; "1" applies to the results with parameter values from Table 1; "2" indicates the modeled results with modified $r_{1}$ and $d_{k}$

Table 2 - parameters of XLPE cable

\begin{tabular}{|l|l|l|l|}
\hline$\rho(\Omega \cdot \mathrm{m})$ & $\rho_{p}(\Omega \cdot \mathrm{m})$ & $r_{1}(\mathrm{~mm})$ & $r_{2}(\mathrm{~mm})$ \\
$2.82 \times 10^{-8}$ & $1.68 \times 10^{-8}$ & 8.6 & 9.4 \\
\hline$r_{4}(\mathrm{~mm})$ & $\theta$ & $d_{k}(\mathrm{~mm})$ & $r_{p 1}(\mathrm{~mm})$ \\
14 & $\pi / 3$ & 16.1 & 29.5 \\
\hline$\varepsilon_{\text {ins }}$ & $\varepsilon_{\text {semi }}[7]$ & $r_{3}(\mathrm{~mm})$ & $r_{p 2}(\mathrm{~mm})$ \\
2.26 & 1000 & 12.8 & 30.5 \\
\hline
\end{tabular}

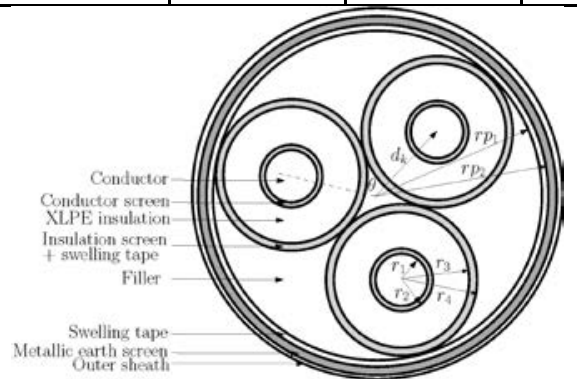

Fig. 3 - Schematic cross-section of XLPE cable 
Since XLPE cable includes a semi-conducting conductor screen and an insulation screen, the model from the PILC cable will be adjusted to incorporate these layers. For the impedance, only the conductor screen can be incorporated into the conductor according to the methodology in [8]. For case of ease, the insulation screen is regarded as part of the insulation. For the potential coefficient, the conductor screen is treated separately while the insulation screen is included in the insulation by adjusting the insulation permittivity [7]. Because of the shielding effect of the semiconducting insulation screen, $\mathbf{P}_{\mathbf{p}}$, which is referred to as pipe internal potential coefficient matrix (potential coefficient between different inner conductors with respect to the common earth screen) [3], is zero.

The measured and modeled characteristic impedance, propagation velocity, attenuation for both PP and SP mode are compared in Fig. 4. Modeled results are comparable with the conformal mapping approach in [5]. The comparison is shown up to $7 \mathrm{MHz}$ since the measured results above this are not reliable due to the lack of energy in the reflected pulses at high frequencies (the chosen pulse has a pulse width of $100 \mathrm{~ns}$ [5]). The deviations of the measured and simulated characteristic impedances are 5\% and 24\% (about $1.7 \Omega$ difference) for the SP and PP mode, respectively. SP mode velocity deviates about $17 \%$ and PP mode $8 \%$. Attenuation calculation is not reliable. The difficulty to include semi-conducting layers, especially the insulation screen in the model could be the cause for the larger error compared with PILC. Furthermore, the large deviations suggest that in this specific situation the modeling approach has its limitations. Basically, the admittance is mainly related to each individual conductor and its insulation screen whereas the series impedance is related to the phases with respect to the outer earth screen or to two phases with respect to each other. Introducing effective parameters for the dimensions, as was done for the PILC configuration, would be quite artificial and is not attempted here.

\section{Conclusions}

A generic approach is evaluated to model the characteristic impedance, propagation velocity, attenuation for PP and SP mode of two different threephase cable configurations: PILC and XLPE cable. For PILC, the measured characteristic impedance varied within $5 \%$ of the simulated value applying an effective conductor radius. The velocity was reproduced within 5\% accuracy, since it is mainly determined by the known real part of the permittivity. The attenuation, although its effect on both series impedance and admittance is accounted for, always suffer from lack of knowledge of the imaginary part of permittivity in practice. The frequency dependent permittivity is kept as constant in this paper, but to be accurate its value should be fitted to match experimental results. For XLPE, due to more complex structure, especially relating to the semiconducting material, estimations are generally worse compared with PILC. The SP mode characteristic impedance deviates $5 \%$ and around $1.7 \Omega$ for PP mode. The velocity deviates about $10 \mathrm{~m} / \mu \mathrm{s}$ for PP mode and $20 \mathrm{~m} / \mu \mathrm{s}$ for SP mode. Modeled attenuation characteristics are far from reliable.
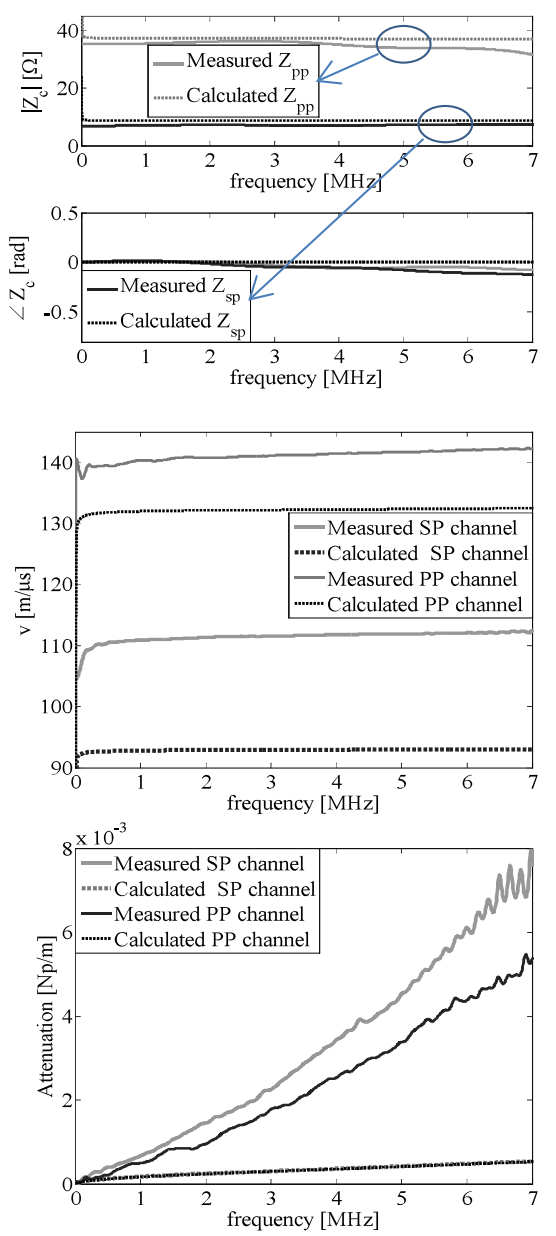

Fig. 4 - Comparison of characteristic impedance, propagation velocity, attenuation for both PP and SP mode for XLPE cable

\section{Appendix}

Detailed analysis based on references [3,4] are given for both PILC and XLPE impedance and potential coefficients.

\subsection{PILC}

$$
\begin{gathered}
\mathbf{Z}_{\mathbf{i}}=\left[\begin{array}{ccc}
Z_{i 1} & 0 & 0 \\
0 & Z_{i 2} & 0 \\
0 & 0 & Z_{i 3}
\end{array}\right] \\
Z_{i 1}=Z_{i 2}=Z_{i 3}=\frac{\rho x}{2 \pi r_{1}^{2}} \frac{I_{0}(x)}{I_{1}(x)}+\frac{j \omega \mu_{0} \mu_{i}}{2 \pi} \ln \left(r_{2} / r_{1}\right)
\end{gathered}
$$

where $\rho$ is the resistivity of the conductor; $\mu_{\mathrm{i}}$ is the permeability for SC cable insulation permeability. $x$ is relating to the skin depth and $x=r_{1} \sqrt{j \omega \mu_{0} \mu_{i} / \rho} ; I_{0,1}$ are the zero and first order modified Bessel function of the first kind and $K_{0,1}$ are the zero and first order modified Bessel function of the second kind; $r_{2}$ is the radius of the SC cable insulation. For the three-core PILC cable 
with common earth screen, $r_{2}=r_{1}$. Thus, the second item of $Z_{\mathrm{i} 1}$ becomes zero.

$$
\begin{aligned}
\mathbf{Z}_{\mathbf{p}}=\left[\begin{array}{lll}
Z_{p 11} & Z_{p 12} & Z_{p 13} \\
Z_{p 12} & Z_{p 22} & Z_{p 23} \\
Z_{p 13} & Z_{p 23} & Z_{p 33}
\end{array}\right] \\
Z_{p 11}=Z_{p 22}=Z_{p 33} \\
=j \omega \frac{\mu_{0}}{2 \pi}\left[\frac{\mu_{p} K_{0}\left(x_{1}\right)}{x_{1} K_{1}\left(x_{1}\right)}+Q_{11}+\right. \\
\left.2 \mu_{p} \sum_{n=1}^{\infty} \frac{C_{n}}{n\left(1+\mu_{p}\right)+x_{1} \frac{K_{n-1}\left(x_{1}\right)}{K_{n}\left(x_{1}\right)}}\right] \\
Z_{p 12}=Z_{p 13}=Z_{p 23} \quad j \omega \frac{\mu_{0}}{2 \pi}\left[\frac{\mu_{p} K_{0}\left(x_{1}\right)}{x_{1} K_{1}\left(x_{1}\right)}+Q_{12}+\right. \\
\left.2 \mu_{p} \sum_{n=1}^{\infty} \frac{C_{n}}{n\left(1+\mu_{p}\right)+x_{1} \frac{K_{n-1}\left(x_{1}\right)}{K_{n}\left(x_{1}\right)}}\right]
\end{aligned}
$$

where

$$
\begin{aligned}
& Q_{11}=\ln \left[\left(r_{p 1} / r_{1}\right)\left(1-d_{k}^{2} / r_{p 1}^{2}\right)\right] \\
& Q_{12}=\ln \left[\left(r_{p 1} / \sqrt{\left.2 d_{k}^{2}-2 d_{k}^{2} \cos \theta\right)}\right]-\sum_{n=1}^{\infty} C_{n} / n\right. \\
& C_{n}=\left(d_{k}^{2} / r_{p 1}^{2}\right)^{n} \cos (n \theta) \\
& x_{1}=r_{p 1} \sqrt{j \omega \mu_{0} \mu_{p} / \rho_{p}}
\end{aligned}
$$

For the potential coefficient:

$$
\begin{aligned}
& \mathbf{P}_{\mathbf{i}}=\left[\begin{array}{ccc}
P_{i 1} & 0 & 0 \\
0 & P_{i 2} & 0 \\
0 & 0 & P_{i 3}
\end{array}\right] \\
& P_{i 1}=P_{i 2}=P_{i 3}=\frac{1}{2 \pi \varepsilon_{0} \varepsilon_{j}} \ln \left(r_{2} / r_{1}\right)
\end{aligned}
$$

where $\varepsilon_{\mathrm{j}}$ is the permittivity of the insulation for an SC cable. Since $r_{2}=r_{1} . \mathbf{P}_{\mathbf{i}}$ becomes a zero matrix.

$$
\mathbf{P}_{\mathbf{p}}=\frac{1}{2 \pi \varepsilon_{0} \varepsilon_{p}}\left[\begin{array}{lll}
Q_{11} & Q_{12} & Q_{12} \\
Q_{12} & Q_{11} & Q_{12} \\
Q_{12} & Q_{12} & Q_{11}
\end{array}\right]
$$

\subsection{XLPE}

Because of the different structure of the XLPE cable, the formulas differ from those for the PILC cable.

$$
\begin{aligned}
\mathbf{Z}_{\mathbf{i}} & =\left[\begin{array}{ccc}
Z_{i 1} & 0 & 0 \\
0 & Z_{i 2} & 0 \\
0 & 0 & Z_{i 3}
\end{array}\right] \\
Z_{i 1}= & Z_{i 2}=Z_{i 3} \\
= & z_{1}+\frac{j \omega \mu_{0} \mu_{i}}{2 \pi} \ln \left(r_{4} / r_{2}\right)
\end{aligned}
$$

where $z_{1}$ is the core impedance incorporating conductor screen; refer to reference [8,9] for detailed formulas. Compared with (6) and (7), the parameters in (15) have the same meaning as for PILC.

For the potential coefficient:

$$
\begin{aligned}
& \mathbf{P}_{\mathbf{i}}=\left[\begin{array}{ccc}
P_{i 1} & 0 & 0 \\
0 & P_{i 2} & 0 \\
0 & 0 & P_{i 3}
\end{array}\right] \\
& P_{i 1}=P_{i 2}=P_{i 3}=P_{\text {semi } 1}+P_{i n s}
\end{aligned}
$$

where

$$
\begin{aligned}
& P_{\text {semi1 }}=\left(1 / 2 \pi \varepsilon_{0} \varepsilon_{\text {semi } 1}\right) \ln \left(r_{2} / r_{1}\right) \\
& P_{\text {ins }}=\left(1 / 2 \pi \varepsilon_{0} \varepsilon_{\text {ins }}\right) \ln \left(r_{4} / r_{2}\right) \\
& \varepsilon_{\text {ins }}{ }^{\prime}=\varepsilon_{\text {ins }} \ln \left(r_{4} / r_{2}\right) / \ln \left(r_{3} / r_{2}\right)
\end{aligned}
$$

\section{References}

[1] Paul Wagenaars, P.A.A.F. Wouters, P.C.J.M. van der Wielen, and E.F. Steennis, "Measurement of transmission line parameters of three-core power cables with common earth screen", IET Science, Measurement \& Technology, vol.4, no.3, May 2010, pp.146-155.

[2] Paul Wagenaars, P.A.A.F. Wouters, P.C.J.M. van der Wielen, and E.F. Steennis, "Approximation of transmission line parameters of single-core and three-core XLPE cables", IEEE Transactions on Dielectrics and Electrical Insulation, vol.17, no.1, February 2010, pp.106-115.

[3] A. Ametani, "A General Formulation of Impedance and Admittance of Cables", IEEE Transactions on Power Apparatus and Systems, vol.PAS-99, no.3, May 1980, pp.902-910.

[4] L.M. Wedepohl, and D.J. Wilcox, "Transient analysis of underground power-transmission systems. System-model and wave-propagation characteristics", Proceedings of the Institution of Electrical Engineers, vol.120, no.2, February 1973, pp.253-260.

[5] Paul Wagenaars, Integration of Online Partial Discharge Monitoring and Defect Location in Medium-Voltage Cable Networks, Ph.D. dissertation, Eindhoven University of Technology, 2010.

[6] P.C.J.M. van der Wielen, On-line Detection and Location of Partial Discharges in Medium-Voltage Power Cables, Ph.D. dissertation, Eindhoven University of Technology, 2005.

[7] B. Gustavsen, "Panel Session on Data for Modeling Insulated Cables", IEEE Power Engineering Society Winter Meeting, 2001, pp.718-723.

[8] Akihiro Ametani, Yukata Miyamoto, and Naoto Nagaoka, "Semiconducting Layer Impedance and its Effect on Cable Wave-Propagation and Transient Characteristics", IEEE Transactions on Power Delivery, vol. 19, no. 4, October 2004, pp.1523-1531.

[9] Y. Li, P.A.A.F. Wouters, P. Wagenaars, P.C.J.M. van der Wielen, and E.F. Steennis, "Estimation of transmission line parameters single-core XLPE cables considering semiconducting layer", Proceedings of the IEEE International Conference on Condition Monitoring and Diagnosis, Indonesia, 23-27 September 2012, pp. 967-970. 